\title{
TWO LIFTING THEOREMS
}

\author{
STUART P. LLOYD
}

\begin{abstract}
It is assumed that the measure algebra involved has cardinality $2 \mathrm{~N}_{0}$, and it is assumed further that $2 \mathrm{~N}_{0}=\mathrm{N}_{1}$. Then liftings exist when the $\sigma$-field is not necessarily complete, and strong Borel liftings exist in the locally compact $\sigma$-compact metric case.
\end{abstract}

1. Introduction. Let $\left(X_{0}, \mathscr{F}_{0}, \mu_{0}\right)$ be a probability space, let $B\left(X_{0}, \mathscr{F}_{0}\right)$ be the Banach algebra of bounded real $\mathscr{F}_{0}$ measurable functions on $X_{0}$, the norm being $\|f\|=\sup _{x}\left\{|f(x)|: x \in X_{0}\right\}$, and let $\mathscr{I}_{0}=\left\{f \in B\left(X_{0}, \mathscr{F}_{0}\right)\right.$ : $\left.\int|f| d \mu_{0}=0\right\}$ be the closed ideal of $\mu_{0}$-null functions. The quotient Banach algebra $B\left(X_{0}, \mathscr{F}_{0}\right) / \mathscr{I}_{0}$ may be identified as the familiar $L_{\infty}\left(X_{0}, \mathscr{F}_{0}, \mu_{0}\right)$; let $q_{0}: B\left(X_{0}, \mathscr{F}_{0}\right) \rightarrow L_{\infty}\left(X_{0}, \mathscr{F}_{0}, \mu_{0}\right)$ be the quotient mapping. A lifting $\Lambda_{0}: L_{\infty}\left(X_{0}, \mathscr{F}_{0}, \mu_{0}\right) \rightarrow B\left(X_{0}, \mathscr{F}_{0}\right)$ is a selection of representative $\Lambda_{0}\left(f+\mathscr{I}_{0}\right) \in$ $f+\mathscr{I}_{0}$ from each equivalence class $f+\mathscr{I}_{0}, f \in B\left(X_{0}, \mathscr{F}_{0}\right)$, in such a way that the representatives constitute a subalgebra of $B\left(X_{0}, \mathscr{F}_{0}\right)$; it is required also that $\Lambda_{0}\left(1+\mathscr{I}_{0}\right)=1$. That is, $\Lambda_{0}$ is an algebraic cross section of $q_{0}$ which preserves the unit: $\Lambda_{0}$ is an algebraic homomorphism, $q_{0} \Lambda_{0}=$ (identity), $\Lambda_{0}\left(1+\mathscr{I}_{0}\right)=1$. The proof in [1] that liftings exist requires that $\mathscr{F}_{0}$ be complete with respect to $\mu_{0}$. We show that $\mathscr{F}_{0}$ need not be complete provided: (i) the measure algebra $\left(\mathscr{F}_{0}, \mu_{0}\right)$ has cardinal $2^{\text {No}_{0}}$; (ii) $2^{\boldsymbol{N}_{0}}=\boldsymbol{N}_{1}$ (the continuum hypothesis).

Suppose further that $X_{0}$ is a topological space, the bounded continuous real functions $C_{b}\left(X_{0}\right)$ are $\mathscr{F}_{0}$ measurable, and $\mu_{0}(U)>0$ for open $U \neq \varnothing$. A strong lifting is a lifting $\Lambda_{0}$ such that $\Lambda_{0}\left(f+\mathscr{I}_{0}\right)=f, f \in C_{b}\left(X_{0}\right)$. Various sufficient conditions are known [1] for the existence of strong liftings, e.g., $X_{0}$ locally compact $\sigma$-compact metric. We prove here (with $2^{\boldsymbol{N}_{0}}=\boldsymbol{N}_{1}$ ) that strong Borel liftings exist in this last case; that is, strong liftings such that each representative is measurable with respect to the uncompleted $\sigma$-field of Borel subsets of locally compact $\sigma$-compact metric $X_{0}$.

2. Representation spaces. Under the Gel'fand representation; $B\left(X_{0}, \mathscr{F}_{0}\right)$ is isometrically algebraically isomorphic to the continuous real functions

Presented to the Society, August 23, 1973; received by the editors January 1, 1973 and, in revised form, April 26, 1973.

AMS (MOS) subject classifications (1970). Primary 46G15.

Key words and phrases. Lifting theory.

(c) American Mathematical Society 1974 
$C(W)$ on a certain compact Hausdorff space $W$; let $\iota: C(W) \rightarrow B\left(X_{0}, \mathscr{F}_{0}\right)$ be the inverse isomorphism. This has adjoint $\iota^{*}: b a\left(X_{0}, \mathscr{F}_{0}\right) \rightarrow \mathrm{rca}(W)$ when we identify the conjugate Banach space $\left[B\left(X_{0}, \mathscr{F}_{0}\right)\right]^{*}$ with the space $b a\left(X_{0}, \mathscr{F}_{0}\right)$ of bounded finitely additive set functions on $\sigma$-field $\mathscr{F}_{0}$, and the conjugate Banach space $[C(W)]^{*}$ with the space $\operatorname{rca}(W)$ of regular Borel measures on $W$. We will assume without essential loss of generality that $B\left(X_{0}, \mathscr{F}_{0}\right)$ separates $X_{0}$, i.e., if $x_{1} \neq x_{2}$ then $f\left(x_{1}\right) \neq f\left(x_{2}\right)$ for some $f \in B\left(X_{0}, \mathscr{F}_{0}\right)$. Then with $\delta_{x}$ the point measure at $x$, the set $\left\{\iota^{*} \delta_{x}: x \in X_{0}\right\}$ constitutes a copy of $X_{0}$ contained as a dense subset of $W \subset w^{*}$-rca $(W)$; we identify $W$ with the set $\left\{\delta_{w}: w \in W\right\} \subset w^{*}$-rca $(W)$ whenever convenient. A bounded real function $f$ on $X_{0} \subset W$ extends to a member of $C(W)$ iff $f \in B\left(X_{0}, \mathscr{F}_{0}\right)$, and $\iota$ is represented as the restriction mapping $\iota g=g \mid X_{0}$, $g \in C(W)$. Each $E \in \mathscr{F}_{0}$ has closure $\mathrm{cl}_{W} E$ which is open, and the correspondence $E \leftrightarrow \mathrm{cl}_{W} E$ is $1-1$ between $\mathscr{F}_{0}$ and the open closed subsets of $W$. The $\sigma$-field of Baire subsets of $W$ is generated by $\left\{\mathrm{cl}_{W} E: E \in \mathscr{F}_{0}\right\}$, and if $\theta \in b a\left(X_{0}, \mathscr{F}_{0}\right)$ is given then $\iota^{*} \theta \in \operatorname{rca}(W)$ is determined on the Baire subsets of $W$ by $\left(\iota^{*} \theta\right)\left(\mathrm{cl}_{W} E\right)=\theta(E), E \in \mathscr{F}_{0}$, and then on the Borel sets by regularity.

As a Banach lattice, $B\left(X_{0}, \mathscr{F}_{0}\right)$ is boundedly $\sigma$-complete $\left(=\boldsymbol{\aleph}_{0}\right.$ reticulated): any countable subset of $B\left(X_{0}, \mathscr{F}_{0}\right)$ bounded above has a supremum in $B\left(X_{0}, \mathscr{F}_{0}\right)$; the isomorphic $C(W)$ enjoys the same property. Dually, $W$ is basically disconnected: disjoint open Baire subsets of $W$ have disjoint open closures [3, Chapter VII]. Equivalently, the interior $F^{\circ}$ of any closed Baire set $F$ is closed, so that a closed Baire set is of the form $\left(\mathrm{cl}_{W} E\right) \cup N$ with $E \in \mathscr{F}_{0}$ and $N$ a closed nowhere dense Baire set. The following results from [2] will be used. If $N \subset W$ is a closed nowhere dense Baire set then $N=\lim _{n} \mathrm{cl}_{W} E_{n}$ for some sequence $E_{1} \supset E_{2} \supset \cdots$ in $\mathscr{F}_{0}$ such that $\lim _{n} E_{n}=\varnothing$. If $\theta \in b a\left(X_{0}, \mathscr{F}_{0}\right)$ is countably additive on $\mathscr{F}_{0}$ then $\left(\iota^{*} \theta\right)(N)=0$ for every closed nowhere dense Baire set $N$.

Let $\mu=\iota^{*} \mu_{0} \in \operatorname{rca}(W)$ correspond to $\mu_{0}$, and let $X$ be the closed support of $\mu$ in $W$. The ideal $\mathscr{I}=\iota^{-1} \mathscr{I}_{0} \subset C(W)$ which corresponds to $\mathscr{I}_{0}$ is clearly $\{f \in C(W): f(X)=0\}$, and the quotient mapping $q_{0}$, isomorphic to the quotient mapping $C(W) \rightarrow C(W) / \mathscr{I}$, is isomorphic to the restriction mapping $q: C(W) \rightarrow C(X)$ given by $q f=f \mid X, f \in C(W)$. Space $X$ is the Gel'fand space of $L_{\infty}\left(X_{0}, \mathscr{F}_{0}, \mu_{0}\right)$, and is hyperstonian with $\mu$ as category measure. That is, $L_{\infty}\left(X_{0}, \mathscr{F}_{0}, \mu_{0}\right)$ is isometrically algebraically isomorphic to $C(X), X$ is extremally disconnected, and $\mu(A)=\mu\left(A^{\circ}\right)>0$ if $A^{\circ} \neq \varnothing$, Borel $A \subset X$. We denote by $\mathscr{F}$ the class of open closed subsets of $X$; the sets $\left\{X \cap \mathrm{cl}_{W} E: E \in \mathscr{F}_{0}\right\}$ (not necessarily distinct) comprise $\mathscr{F}$. The measure algebra $\left(\mathscr{F}_{0}, \mu_{0}\right)$ is isomorphic to the quotient Boolean algebra $\mathscr{F} /$ (nowhere dense sets). 
The following relation between the closure operators in $W$ and $X$ is needed in the proof of Theorem 1 .

LEMMA 1. If $U \subset W$ is an open Baire set then $X \cap \mathrm{cl}_{W} U=\mathrm{cl}_{X}(X \cap U)$.

Proof: We have $\Phi=\mathrm{cl}_{W} U=U \cup N$ with $\Phi$ open closed and $N$ a closed nowhere dense Baire set in $W$. Since $\mu(X \cap N)=0$ and $\mu$ is category relative to $X, X \cap N$ is nowhere dense in $X$. The closure $\Theta=\operatorname{cl}_{X}(X \cap U)=$ $(X \cap U) \cup N_{1}$ of open $X \cap U$ is open closed in extremally disconnected $X$. Thus

$$
\Theta=[X \cap(\Phi-N)] \cup N_{1}=(X \cap \Phi) \Delta\left[(X \cap N)-N_{1}\right],
$$

and since $\Theta$ and $X \cap \Phi$ are each open closed and $X \cap N$ is nowhere dense, $\Theta=X \cap \Phi$.

3. Partial liftings. To a lifting $\Lambda_{0}: L_{\infty}\left(X_{0}, \mathscr{F}_{0}, \mu_{0}\right) \rightarrow B\left(X_{0}, \mathscr{F}_{0}\right)$ there corresponds an algebraic homomorphism $\Lambda: C(X) \rightarrow C(W)$ with the properties $q \Lambda=$ (identity), $\Lambda 1=1$; we call $\Lambda$ a lifting also. The adjoint $\Lambda^{*}: \operatorname{rca}(W) \rightarrow \operatorname{rca}(X)$ restricts to a mapping $\lambda: W \rightarrow X$ which is a retraction of $W$ onto $X$. Conversely, such a retraction determines a lifting according to: $(\Lambda f)(w)=f(\lambda w), w \in W, f \in C(X)$.

We denote by $\mathscr{A}$ the set $\mathscr{A}=\{\alpha \subset C(X): \alpha$ is a closed subalgebra of $C(X)$ containing the constants $\}$. Each $\alpha \in \mathscr{A}$ is isometrically algebraically isomorphic to $C\left(Z_{\alpha}\right)$ for a certain compact Hausdorff space $Z_{\alpha}$. If $j_{\alpha}$ : $C\left(Z_{\alpha}\right) \rightarrow C(X)$ is the injection onto $\alpha$ then $j_{\alpha}^{*}: \operatorname{rca}(X) \rightarrow \operatorname{rca}\left(Z_{\alpha}\right)$ restricts to the quotient mapping $v_{\alpha}: X \rightarrow Z_{\alpha}$ associated with $\alpha$, i.e., $\left(j_{\alpha} f\right)(x)=f\left(v_{\alpha} x\right)$, $x \in X, f \in C\left(Z_{\alpha}\right)$.

By a partial lifting $\Lambda_{\alpha}: \alpha \rightarrow C(W)$ we will mean an algebraic homomorphism defined only on the subalgebra $\alpha$ of $C(X)$ with the properties $q \Lambda_{\alpha}=$ (identity), $\Lambda_{\alpha} 1=1$. Equivalent to $\Lambda_{\alpha}$ is the algebraic homomorphism $\tilde{\Lambda}_{\alpha}: C\left(Z_{\alpha}\right) \rightarrow C(W)$ given by $\tilde{\Lambda}_{\alpha}=\Lambda_{\alpha} j_{\alpha}$ and such that $q \tilde{\Lambda}_{\alpha}=j_{\alpha}, \tilde{\Lambda}_{\alpha} 1=1$. The adjoint $\tilde{\Lambda}_{\alpha}^{*}: \operatorname{rca}(W) \rightarrow \operatorname{rca}\left(Z_{\alpha}\right)$ restricts to the partial retraction $\lambda_{\alpha}: W \rightarrow Z_{\alpha}$ dual to $\tilde{\Lambda}_{\alpha}$ and $\Lambda_{\alpha}$, i.e., $\lambda_{\alpha} \mid X=v_{\alpha}$. Since $\tilde{\Lambda}_{\alpha}$ and $j_{\alpha}^{-1}$ are isometries, $\Lambda_{\alpha}=\tilde{\Lambda}_{\alpha} j_{\alpha}^{-1}$ is an isometry.

Let $\mathscr{L}$ denote the family $\mathscr{L}=\left\{\Lambda_{\alpha}: \alpha \in \mathscr{A}\right.$ and $\Lambda_{\alpha}$ is a partial lifting with domain $\alpha$ \}. An order $<$ in $\mathscr{L}$ is defined by: $\Lambda_{\alpha}<\Lambda_{\beta}$ iff $\Lambda_{\beta}$ extends $\Lambda_{\alpha}$; that is, $\alpha \subset \beta$ and $\Lambda_{\beta} \mid \alpha=\Lambda_{\alpha}$.

LEMMA 2. Any ascending chain in $\mathscr{L}$ has an upper bound in $\mathscr{L}$.

Proof. Suppose $\left\{\Lambda_{\alpha_{v}}: \nu \in M\right\}$ is an ascending chain in $\mathscr{L}: M$ is a totally ordered indexing set and $\Lambda_{\alpha_{\mu}}<\Lambda_{\alpha_{\nu}}$ for $\mu \leqq \nu \in M$. Define subalgebra $\gamma$ of $C(X)$ as $\gamma=\bigcup_{\nu}\left\{\alpha_{v}: v \in M\right\}$, and let $\alpha \in \mathscr{A}$ be the closure in $C(X)$ of $\gamma$. An operator $\Lambda_{\gamma}: \gamma \rightarrow C(W)$ is defined consistently on $\gamma$ by the family 
of its restrictions $\Lambda_{\gamma} \mid \alpha_{\mu}=\Lambda_{\alpha \mu}, \mu \in M$, and one verifies easily that $\Lambda_{\gamma}$ is an algebraic homomorphism with the properties $q \Lambda_{\gamma}=$ (identity), $\Lambda_{\gamma} 1=1$. Since each $\Lambda_{\alpha \mu}, \mu \in M$, is an isometry, $\Lambda_{\gamma}$ is an isometry, and so extends uniquely by continuity to an operator $\Lambda_{\alpha}: \alpha \rightarrow C(W)$. By continuity, $\Lambda_{\alpha}$ is an algebraic homomorphism with the properties $q \Lambda_{\alpha}=$ (identity), $\Lambda_{\alpha} 1=1$; that is, $\Lambda_{\alpha} \in \mathscr{L}$. Since $\Lambda_{\alpha \mu}<\Lambda_{\alpha}, \mu \in M$, by construction, $\Lambda_{\alpha}$ is the upper bound sought.

Suppose $\alpha \in \mathscr{A}$ and $X_{1} \in \mathscr{F}$ are given, and let $\beta \in \mathscr{A}$ be the algebra generated by $\left\{\alpha, \chi_{X_{1}}\right\}$. With $X_{2}=X-X_{1}, \beta$ consists of all $f \in C(X)$ of the form $f=f_{1} \chi_{X_{1}}+f_{2} \chi_{X_{2}}$ for some $f_{1}, f_{2} \in \alpha$. We may rewrite this as $f=\left(f_{1}+\mathscr{I}_{1}\right) \chi_{X_{1}}+\left(f_{2}+\mathscr{I}_{2}\right) \chi_{X_{2}}$ where $\mathscr{I}_{i}=\left\{g \in \alpha: g \chi_{X_{i}}=0\right\}, i=1,2$, are closed ideals in $\alpha$; the elements $\left(f_{i}+\mathscr{I}_{i}\right) \in \alpha / \mathscr{I}_{i}, i=1,2$, are then uniquely determined by $f \in \beta$. The space $Z_{\beta}$ associated with $\beta$ is the free union $Z_{\beta}=Z_{\alpha 1} \cup Z_{\alpha 2}$ of copies of the subsets $Z_{\alpha i}=v_{\alpha} X_{i}$, with $\alpha / \mathscr{I}_{i}$ isomorphic to $C\left(Z_{\alpha i}\right), i=1,2$.

Lemma 3. Suppose $\Lambda_{\alpha} \in \mathscr{L}$ and $X_{1}, X_{2}=X-X_{1} \in \mathscr{F}$ are given, and let $\beta \in \mathscr{A}$ be the algebra generated by $\left\{\alpha, \chi_{x_{1}}\right\}$. If $\Lambda_{\beta} \in \mathscr{L}$ exists such that $\Lambda_{\alpha}<\Lambda_{\beta}$ then open closed subsets $W_{1}$ and $W_{2}=W-W_{1}$ of $W$ are determined such that

(i) $\Lambda_{\beta} f=\left(\Lambda_{\alpha} f_{1}\right) \chi_{W_{1}}+\left(\Lambda_{\alpha} f_{2}\right) \chi_{w_{2}}$ for $f=\left(f_{1}+\mathscr{I}_{1}\right) \chi_{X_{1}}+\left(f_{2}+\mathscr{I}_{2}\right) \chi_{X_{2}} \in \beta$,

(ii) $W_{i} \cap X=X_{i}, i=1,2$,

(iii) $W_{i} \subset \lambda_{\alpha}^{-1} v_{\alpha} X_{i}, i=1,2$.

Conversely, if open closed $W_{1}$ and $W_{2}=W-W_{1}$ in $W$ are given satisfying (ii) and (iii) then (i) serves to define $\Lambda_{\beta} \in \mathscr{L}$ such that $\Lambda_{\alpha}<\Lambda_{\beta}$.

Proof. Suppose $\Lambda_{\beta} \in \mathscr{L}$ is given such that $\Lambda_{\alpha}<\Lambda_{\beta}$. From $\chi_{X_{1}}^{2}=\chi_{X}$ and $\chi_{X_{2}}=1-\chi_{X_{1}}$, and the fact that $\Lambda_{\beta}$ is an algebraic homomorphism such that $\Lambda_{\beta} 1=1$, we find that $\Lambda_{\beta} \chi_{X_{i}}=\chi_{W_{i}}, i=1,2$, for open closed subsets $W_{1}$ and $W_{2}=W-W_{1}$ of $W$. Since $\mathscr{I}_{i} \subset \alpha, \chi_{X_{i}} \in \beta$, and $\mathscr{I}_{i} \chi_{X_{i}}=0$, $i=1,2$, we must have

$$
\Lambda_{\beta}\left(\mathscr{I}_{i} \chi_{X_{i}}\right)=\left(\Lambda_{\alpha} \mathscr{I}_{i}\right)\left(\Lambda_{\beta} \chi_{X_{i}}\right)=\left(\Lambda_{\alpha} \mathscr{I}_{i}\right) \chi_{W_{i}}=0, \quad i=1,2 ;
$$

this is condition (iii). The property $q \Lambda_{\beta}=$ (identity) gives condition (ii). The converse arguments are similar.

Theorem 1. Suppose $\Lambda_{\alpha} \in \mathscr{L}$ and $X_{1} \in \mathscr{F}$ are given, and let $\beta \in \mathscr{A}$ be the subalgebra generated by $\left\{\alpha, \chi_{X_{1}}\right\}$. If $Z_{\alpha}$ is metrizable then partial liftings $\Lambda_{\beta}$ exist which extend $\Lambda_{\alpha}$.

Proof. The conditions $W_{i} \subset \lambda_{\alpha}^{-1} v_{\alpha} X_{i}, i=1,2$, of Lemma 3 are equivalent to $W_{i} \supset \lambda_{\alpha}^{-1} U_{i}, i=1,2$, where $U_{i}=Z_{\alpha}-v_{\alpha} X_{3-i}, i=1,2$, are disjoint open subsets of $Z_{\alpha}$. If $Z_{\alpha}$ is metrizable the Borel sets are Baire sets, $U_{1}$ and $U_{2}$ are disjoint open Baire sets in $Z_{\alpha}$, whence $\lambda_{\alpha}^{-1} U_{1}$ and $\lambda_{\alpha}^{-1} U_{2}$ are disjoint 
open Baire sets in $W$. Using the fact that $W$ is basically disconnected, we have that $\Phi_{1}=\mathrm{cl}_{W}\left(\lambda^{-1} U_{1}\right)$ and $\Phi_{2}=\mathrm{cl}_{W}\left(\lambda^{-1} U_{2}\right)$ are disjoint open closed subsets of $W$.

By Lemma 1,

$$
X \cap \Phi_{1}=\operatorname{cl}_{X}\left(X \cap \lambda_{\alpha}^{-1} U_{1}\right)=\operatorname{cl}_{X}\left(v_{\alpha}^{-1} U_{1}\right)=\operatorname{cl}_{X}\left(X-v_{\alpha}^{-1} v_{\alpha} X_{2}\right) \subset X_{1},
$$

since $X-v_{\alpha}^{-1} v_{\alpha} X_{2} \subset X_{1}$ and $X_{1}$ is closed; similarly, $\Phi_{2} \cap X \subset X_{2}$.

Let $\Gamma_{1}$ and $\Gamma_{2}=W-\Gamma_{1}$ be any open closed subsets of $W$ such that $X \cap \Gamma_{i}=X_{i}, \quad i=1,2$. With $\Theta=W-\left(\Phi_{1} \cup \Phi_{2}\right)$ open closed, define open closed $W_{1}$ and $W_{2}$ by $W_{i}=\Phi_{i} \cup\left(\Theta \cap \Gamma_{i}\right), i=1,2$. It is clear that $W_{2}=W-$ $W_{1}$. Since $W_{i} \cap X=\left(\Phi_{i} \cap X\right) \cup\left(\Theta \cap X_{i}\right) \subset X_{i}$ and $\left\{W_{1}, W_{2}\right\},\left\{X_{1}, X_{2}\right\}$ are partitions, we have necessarily $W_{i} \cap X=X_{i}, i=1,2$. Conditions (ii) and (iii) of Lemma 3 being satisfied, (i) gives the extension sought.

4. The Lifting theorems. The cardinal of the measure algebra $\left(\mathscr{F}_{0}, \mu_{0}\right)$ is either finite or at least $2^{\mathrm{x}_{0}}$; we assume from now on that the cardinal is $2^{\boldsymbol{N}_{0}}$. We assume further that $2^{\boldsymbol{N}_{0}}=\boldsymbol{\aleph}_{1}$, and we let $\left\{F_{v}: \nu<\boldsymbol{K}_{1}\right\}$ be a well ordering of the elements of $\mathscr{F}$.

THEOREM 2 (INCOMPLETE LIFTING THEOREM). If the measure algebra $\left(\mathscr{F}_{0}, \mu_{0}\right)$ has cardinal $2^{\aleph_{0}}=\aleph_{1}$ then liftings $\Lambda_{0}: L_{\infty}\left(X_{0}, \mathscr{F}_{0}, \mu_{0}\right) \rightarrow B\left(X_{0}, \mathscr{F}_{0}\right)$ exist.

Proof. The parts of the transfinite induction are:

(i) $\alpha_{0}$ is the constants, $Z_{\alpha_{0}}$ is a singleton, $\Lambda_{\alpha_{0}} 1=1$.

(ii) For $\nu<\mathcal{K}_{1}$ a successor ordinal, suppose $\left\{\Lambda_{\alpha \gamma}: \gamma<\nu\right\}$ is an ascending chain in $\mathscr{L}$ such that each $Z_{\alpha \gamma}, \gamma<\nu$, is metrizable; in particular, $Z_{\alpha_{\nu-1}}$ is metrizable. Let $\alpha_{v}$ be the algebra generated by $\left\{\alpha_{v-1}, \chi_{F_{v-1}}\right\}$, and let $\Lambda_{\alpha_{v}}$ be the partial lifting provided by Theorem 1 . It is clear that $Z_{\alpha_{v}}$ is metrizable, so that $\left\{\Lambda_{\alpha \gamma}: \gamma<\nu+1\right\}$ is an ascending chain in $\mathscr{L}$ such that each $Z_{\alpha \gamma}$ is metrizable, $\gamma<\nu+1$.

(iii) For $\nu<\aleph_{1}$ a limit ordinal, suppose $\left\{\Lambda_{\alpha \gamma}: \gamma<\nu\right\}$ is an ascending chain in $\mathscr{L}$ such that each $Z_{\alpha \gamma}, \gamma<\nu$, is metrizable. Lemma 2 provides $\Lambda_{\alpha \nu}$ on $\alpha_{\nu}=\operatorname{cl}_{C(X)} \bigcup_{\gamma<\nu} \alpha_{\gamma}$ such that $\left\{\Lambda_{\alpha \gamma}: \gamma<\nu+1\right\}$ is an ascending chain in $\mathscr{L}$. If $\sigma_{\gamma} \subset \alpha_{\gamma}$ is a countable set dense in $\alpha_{\gamma}, \gamma<\nu$, then $\bigcup_{\gamma<\nu} \sigma_{\gamma} \subset \alpha_{\nu}$ is a countable set dense in $\alpha_{\nu}$, so that $Z_{\alpha_{\nu}}$ is metrizable.

By transfinite induction, there exists an ascending chain $\left\{\Lambda_{\alpha \gamma}: \gamma<\boldsymbol{N}_{1}\right\}$, and Lemma 2 provides an ascending chain $\left\{\Lambda_{\alpha \gamma}: \gamma \leqq \aleph_{1}\right\}$. The algebra $\alpha_{\aleph_{1}}=\bigcup_{\nu<\aleph_{1}} \alpha_{v} \in \mathscr{A}$ contains every $\chi_{F_{v}}, \nu<\aleph_{1}$, and so is all of $C(X)$. Thus the partial lifting $\Lambda_{\alpha_{N_{1}}}$ is a lifting.

TheOREM 3 (Strong Borel LifTING THEOREM). Let $X_{0}$ be a locally compact $\sigma$-compact metric space, let $\mathscr{F}_{0}$ be the Borel subsets of $X_{0}$, let $\mu_{0}$ be strictly positive on nonempty open sets, and assume $2^{\mathrm{N}_{0}}=\aleph_{1}$. Then 
liftings $\Lambda_{0}: L_{\infty}\left(X_{0}, \mathscr{F}_{0}, \mu_{0}\right) \rightarrow B\left(X_{0}, \mathscr{F}_{0}\right)$ exist such that $\Lambda_{0}\left(f+\mathscr{I}_{0}\right)=f$, $f \in C_{b}\left(X_{0}\right)$.

Proof. With $C_{0}\left(X_{0}\right)$ the continuous real functions vanishing at infinity, let $A_{0} \subset C_{b}\left(X_{0}\right)$ be the algebra generated by $\left\{C_{0}\left(X_{0}\right), 1\right\} ; A_{0}$ is isometrically algebraically isomorphic to $C\left(Z_{\alpha_{0}}\right)$ where $Z_{\alpha_{0}}=X_{0} \cup\{\infty\}$ is the one point compactification of $X_{0}$ if $X_{0}$ is noncompact, or $Z_{\alpha_{0}}=X_{0}$ if $X_{0}$ is compact. The assumption that $\mu_{0}$ is strictly positive on nonempty open sets implies that for each $f \in A_{0}, f$ is the unique continuous function in the class $f+\mathscr{I}_{0} \in L_{\infty}\left(X_{0}, \mathscr{F}_{0}, \mu_{0}\right)$. Equivalently, a partial lifting $\Lambda_{\alpha_{0}}: \alpha_{0} \rightarrow C(W)$ of the subalgebra $\alpha_{0}=q \iota^{-1} A_{0} \subset C(X)$ is determined such that $\Lambda_{\alpha_{0}} q \iota^{-1} f=\iota^{-1} f$, $f \in A_{0}$. The space $Z_{\alpha_{0}}$ associated with $\alpha_{0}$ is the one defined above, and the assumption that $X_{0}$ is $\sigma$-compact implies that $Z_{\alpha_{0}}$ is metrizable.

We now apply transfinite induction; parts (ii) and (iii) are as in the proof of Theorem 2, but part (i) is: $\alpha_{0}=q \imath^{-1} A_{0}, Z_{\alpha_{0}}$ and $\Lambda_{\alpha_{0}}$ as just described. We obtain a lifting $\Lambda: C(X) \rightarrow C(W)$ such that $\Lambda_{x_{0}}<\Lambda$; the isomorphic $\Lambda_{0}: L_{\infty}\left(X_{0}, \mathscr{F}_{0}, \mu_{0}\right) \rightarrow B\left(X_{0}, \mathscr{F}_{0}\right)$ is such that $\Lambda_{0}\left(f+\mathscr{I}_{0}\right)=f, f \in A_{0}$.

If $X_{0}$ is compact we are done; suppose $X_{0}$ is noncompact. Since $X_{0}$ is assumed to be $\sigma$-compact, there exists $h \in C_{0}\left(X_{0}\right)$ such that $h(x)>0$, $x \in X_{0}$. From

$$
\Lambda_{0}\left(h f+\mathscr{I}_{0}\right)=\left[\Lambda_{0}\left(h+\mathscr{I}_{0}\right)\right]\left[\Lambda_{0}\left(f+\mathscr{I}_{0}\right)\right], \quad f \in B\left(X_{0}, \mathscr{F}_{0}\right),
$$

and $\Lambda_{0}\left(h+\mathscr{I}_{0}\right)=h>0$ we have

$$
\Lambda_{0}\left(f+\mathscr{I}_{0}\right)=h^{-1} \Lambda_{0}\left(h f+\mathscr{I}_{0}\right), \quad f \in B\left(X_{0}, \mathscr{F}_{0}\right) .
$$

If $f \in C_{b}\left(X_{0}\right)$ then $h f \in C_{0}\left(X_{0}\right)$ and $\Lambda_{0}\left(h f+\mathscr{I}_{0}\right)=h f$, giving $\Lambda_{0}\left(f+\mathscr{I}_{0}\right)=f$, $f \in C_{b}\left(X_{0}\right)$. That is, $\Lambda_{0}$ is a strong Borel lifting.

We conclude with the following remarks. In the proof of the lifting theorem given in [1] it is required that the subalgebras $\alpha$ involved in the partial liftings be boundedly complete; that is, the $Z_{x}$ are extremally disconnected. In the induction step corresponding to Theorem 1 of the present paper the sets $U_{1}, U_{2} \subset Z_{\alpha}$ have closures in $Z_{\alpha}$ which are disjoint and open closed, hence Baire, and these closures can replace $U_{1}, U_{2}$ in the argument. The induction step corresponding to Lemma 2 becomes much more difficult, however. The partial liftings $\Lambda_{\alpha \nu}, \nu \in M$, must be extended not only to our $\alpha=\operatorname{cl}_{C(X)}\left[\bigcup\left\{\alpha_{\nu}: v \in M\right\}\right]$ (this is the elementary $L_{\infty}$ martingale theorem given above) but to the boundedly complete algebra generated by $\alpha$; this requires the completeness of $\mathscr{F}_{0}$ with respect to $\mu_{0}$ [1, Theorem IV. 2].

ADDED IN PROOF. Theorem 2 of the present paper, but not Theorem 3 , can be derived from the results of [4]. 


\section{REFERENCES}

1. A. Ionescu Tulcea and C. Ionescu Tulcea, Topics in the theory of lifting, Ergebnisse der Math. und ihrer Grenzgebiete, Band 48, Springer-Verlag, New York, 1969. MR 43 \#2185.

2. S. P. Lloyd, On finitely additive set functions, Proc. Amer. Math. Soc. 14 (1963), 701-704. MR 28 \#4071.

3. Zbigniew Semadeni, Banach spaces of continuous functions. I, PWN, Warsaw, 1971.

4. J. von Neumann and M. H. Stone, The determination of representative elements in the residual classes of a Boolean algebra, Fund. Math. 25 (1935), 353-378.

Mathematics and Statistics Research Center, Bell Telephone laboratories, InC., Murray Hill, New Jersey 07974 